\title{
Simultaneous determination of $\beta$-alanine betaine and trimethylamine in bacterial culture and plant samples by capillary electrophoresis
}

\author{
Isam A Mohamed Ahmed ${ }^{1,2^{*}}$, Ailijiang Maimaiti ${ }^{2}$, Nobuhiro Mori ${ }^{3}$, Norikazu Yamanaka ${ }^{2}$ and Takeshi Taniguchi ${ }^{2}$
}

\begin{abstract}
Background: 3-N-trimethylaminopropionic acid ( $\beta$-alanine betaine) and trimethylamine (TMA) are important nitrogenous compounds that perform fundamental roles in biological pathways throughout all kingdoms of life; however, yet their simultaneous determination method is hardly reported.

Methods: Capillary electrophoresis method for the simultaneous determination of TMA and $\beta$-alanine betaine in microbial culture and plant samples was developed. To increase the sensitivity, TMA and $\beta$-alanine betaine in the samples were first derivatized with bromophenacyl bromide and then analyzed by capillary electrophoresis under low pH.

Results: The derivatization was found to be practically useful for the elimination of interfering substances from plant and microbial extracts, as well as giving well resolved peaks for the analytes ( $\beta$-alanine betaine esters and TMA salt). Analytical features of the developed method showed its respectable performance in terms of linearity $\left(r^{2}>0.99\right)$, precision (relative standard deviation $\left.(\mathrm{RSD})<5 \%\right)$, and detection limits $(0.01 \mathrm{mM})$.

Conclusion: The developed method allows the quantitative determination of TMA and $\beta$-alanine betaine in complex biological samples and assists to study biosynthetic and degradation pathways of these important compounds.
\end{abstract}

Keywords: $\beta$-alanine betaine; Capillary electrophoresis; Microbial culture; Plant leaves; Trimethylamine

\section{Background}

In nature, many plants, bacteria, and marine algae accumulate quaternary ammonium compounds in response to various environmental stresses such as flooding, freezing, heating, drought, and salinity (Rhodes and Hanson 1993; Gorham 1995). These compounds form a structurally heterogeneous class of compounds with a unifying character of a polar and fully methyl substituted nitrogen atom, creating a permanent positive charge on the $\mathrm{N}$ moiety (Rhodes and Hanson 1993). Of them, glycine betaine, 3- $\mathrm{N}$-trimethylaminopropionic acid ( $\beta$-alanine betaine), and proline betaine are known to be the most effective osmoprotectants and are widely distributed in the biosphere (Yancey 2005). Although glycine betaine is extensively

\footnotetext{
* Correspondence: isamnawa@yahoo.com

'Department of Food Science and Technology, Faculty of Agriculture,

University of Khartoum, Shambat 13314, Sudan

${ }^{2}$ Arid Land Research Center, Tottori University, 1390 Hamasaka, Tottori

680-0001, Japan

Full list of author information is available at the end of the article
}

accumulated in many plants in response to various environmental stresses, various members of the highly stresstolerant plant family Plumbaginaceae accumulate $\beta$-alanine betaine instead of glycine betaine (Hanson et al. 1994). $\beta$-Alanine betaine synthesis is not controlled by choline availability, because it is derived from $\beta$-alanine by threestep methylation (Rathinasabapathi et al. 2000). Distinct from glycine betaine synthesis, $\beta$-alanine betaine synthesis does not require oxygen, and therefore, it was proposed to be suitable for osmoprotection under saline and hypoxic conditions (Hanson et al. 1994; Rathinasabapathi et al. 2000). Consequently, $\beta$-Alanine betaine appears to be effective over a broader ecological spectrum than glycine betaine (Rhodes and Hanson 1993).

In soil microorganisms, our recent reports revealed that $\beta$-alanine betaine was accumulated as an intermediate metabolite in the degradation pathway of homocholine by members of the genera Arthrobacter, Rhodococcus, and Pseudomonas (Mohamed Ahmed et al. 2009a, b; Mohamed 
Ahmed et al. 2010; Mohamed Ahmed et al. 2014). The potential role of $\beta$-alanine betaine in plants' and microorganisms' tolerance to salinity and hypoxia makes its synthetic pathway an interesting target for metabolic engineering. However, the estimation methods of this interesting metabolite are still scarce.

Trimethylamine (TMA) is a volatile low molecular weight tertiary aliphatic amine that has been recognized widely in many animal and plant tissues and is one of the degradation products of nitrogenous organic material such as quaternary ammonium compounds such as choline and homocholine (Craciun and Balskus 2012; Mohamed Ahmed et al. 2010). Commonly, the amount of TMA is a useful indicator of spoilage in fresh and lightly preserved seafood as it increases during the breakdown of seafood, such as fish and shrimp (Dalgaard 2006; Ghaly et al. 2010). In medical diagnosis, an increase in the concentration of TMA in the breath of patients can be used as a sign of viremic disease (Siminhoff et al. 1977). Therefore, detection of TMA is of high interests in many fields such environmental protection, food industry, and medical diagnosis. However, one of the challenging aspects of the analysis of $\beta$-alanine betaine and TMA lies in their lack of useful chromospheres, and their chemical structures have permanently charged groups that prevent gas chromatographic separation in their intact forms. In the past, analyses of $\beta$-alanine betaine and TMA relied on qualitative or semi-quantitative colorimetric tests that employed either thin-layer chromatography and Dragendorff's reagent or reaction with picric acid to form colored complex (Blunden et al. 1981; Grieve and Grattan 1983). Since these methods are limited in their sensitivity, selectivity and quantitative accuracy, and ability to assay betaines and TMA in one sample, knowledge of the identities and absolute concentrations of $\beta$-alanine betaine and TMA in biological materials remained inadequate. Recently, capillary electrophoresis has been applied in many different fields because of its extremely high resolution, its speed, and its applicability to a wide range of molecules whether they are charged or uncharged, or of low or high molecular weight (Shintani and Polonsky 1997). In the present work, capillary electrophoresis method under low pH (Nishimura et al. 2001; Zhang et al. 2002) was effectively improved for simultaneous determination of $\beta$-alanine betaine and TMA in both plant leaves and microbial culture samples.

\section{Methods}

\section{Materials}

$\beta$-alanine betaine was synthesized by $N$-methylation of dimethylaminopropionic acid (Tokyo Kasei Kogyo Co. Ltd, Tokyo, Japan) with methyl iodide as described previously (Mohamed Ahmed et al. 2010). Briefly, $4 \mathrm{ml}$ of methyl iodide was added to a suspension of dimethylaminopropionic acid $(1 \mathrm{~g}, 6.5 \mathrm{mM})$ and $\mathrm{KHCO}_{3}(1.3 \mathrm{~g}, 13 \mathrm{mM})$ in $20 \mathrm{ml}$ of methanol. The mixture was stirred overnight at room temperature and then decanted. Thereafter, the liquid phase was concentrated, and the residue was extracted using $15 \mathrm{ml}$ of mixed solvent (acetonitrile/methanol = 10:1, $v / v)$. The combined extracts were dried under a nitrogen stream to give $\beta$-alanine betaine as a colorless powder $(1.2 \mathrm{~g}, 63.2 \%)$. The structure and purity of $\beta$-alanine betaine were confirmed using proton nuclear magnetic resonance $\left({ }^{1} \mathrm{H}\right.$ NMR) and capillary electrophoresis. Unless otherwise specified, all other reagents were of analytical grade and were from either Wako (Wako Pure Chemical Industries Ltd, Tokyo, Japan) or Sigma (St. Louis, MO, USA).

\section{Extract preparation from microbial samples}

Homocholine-degrading strains were isolated from the soil samples obtained from different locations at Tottori University and around Tottori City, Japan. The bacterial strains were cultivated for $24 \mathrm{~h}$ at $30^{\circ} \mathrm{C}$ on $75 \mathrm{ml}$ of basal homocholine liquid media containing $20 \mathrm{mM}$ homocholine as a sole source of carbon, nitrogen, and energy. The cells were harvested at the exponential phase by centrifugation at $10,000 \times g$ for $20 \mathrm{~min}$ at $4^{\circ} \mathrm{C}$. The supernatant was collected and preserved at $-20^{\circ} \mathrm{C}$ until used for detection of $\beta$-alanine betaine and TMA. The harvested bacterial cells were washed three times with saline solution $(8.5 \mathrm{~g} / \mathrm{l} \mathrm{KCl})$, and re-suspended in $50 \mathrm{mM}$ potassium phosphate buffer ( $\mathrm{pH} 7.5)$. The resting cell reaction was started by the addition of homocholine $(20 \mathrm{mM})$ to the cell suspension. The suspension was incubated on a shaker at $120 \mathrm{rpm}$ and $30^{\circ} \mathrm{C}$. At appropriate time intervals ( $30 \mathrm{~min}, 1 \mathrm{~h}, 2 \mathrm{~h}, 3 \mathrm{~h}$, and $6 \mathrm{~h}$ ), aliquots of the cell suspension were withdrawn and boiled for 3 to $5 \mathrm{~min}$ to stop the reaction. These extracts were preserved at $-20^{\circ} \mathrm{C}$ until used for sample derivatization.

\section{Extract preparation from plant samples}

Plant (Limonium suffruticosum, Phragmites australis, and Elaeagnus oxycarpa) leaf samples, at productive stage, were collected from an area around Aiding Lake in the Turpan Basin, Xinjiang, China, in August 2010. The area of the study site is about $10,000 \mathrm{~m}^{2}(100 \mathrm{~m} \times 100 \mathrm{~m})$, and three plots $(10 \mathrm{~m} \times 10 \mathrm{~m})$ were established randomly. The samples were collected from five plants of each species and carefully washed with water. The samples were dried in oven at $85^{\circ} \mathrm{C}$ for $48 \mathrm{~h}$, ground to fine power, and then brought to Arid Land Research Center, Tottori University, Japan, for analysis. For extract preparation, about $100 \mathrm{mg}$ of powdered samples were added to $1.5 \mathrm{ml}$ water, mixed in a plastic tube, incubated at $75^{\circ} \mathrm{C}$ for $20 \mathrm{~min}$, and then centrifuged at $15,000 \times g$ for $10 \mathrm{~min}$. These samples were preserved at $-20^{\circ} \mathrm{C}$ until used for sample derivatization. 


\section{Sample derivatization}

One of the challenging aspects of analysis of $\beta$-alanine betaine and TMA lies in their lack of useful chromophores and thus could not be detected in ultravioletvisible (UV/vis) light range. To overcome this limitation, the samples were derivatized with 4-bromphencyl bromide before analysis with capillary electrophoresis. Esterification was carried out following the methods of Nishimura et al. (2001) with some modifications. Briefly, $0.1 \mathrm{ml}$ of the sample extract and/or authentic standards of $\beta$-alanine betaine and TMA were placed in a microtube and mixed with $0.05 \mathrm{ml}$ of buffer solution $(100 \mathrm{mM}$ $\mathrm{KH}_{2} \mathrm{PO}_{4} /$ distilled water/acetonitrile $\left.=1: 1: 4\right)$. To the mixture, $0.3 \mathrm{ml}$ of 4-bromophenacyl bromide $(20 \mathrm{mg} / \mathrm{ml}$ in acetonitrile) was added. The tube was capped and heated at $90^{\circ} \mathrm{C}$ for $90 \mathrm{~min}$. The reaction mixture was evaporated to dryness with a centrifugal evaporator (CVE-200D; Tokyo Rikakikai, Tokyo, Japan). The residue was dissolved in $300 \mu$ of $50 \mathrm{mM}$ sodium phosphate buffer $(\mathrm{pH} 3.0)$, mixed well, and centrifuged at $10,000 \times g$ for $20 \mathrm{~min}$ at $4^{\circ} \mathrm{C}$. The supernatants, which contained ester and salt of the metabolites $\beta$-alanine betaine and TMA, were filtered using 45- $\mu \mathrm{m}$ filter (Millex Millipore, Billerica, MA, USA) to remove the micro-particles that might block the flow through the capillary tube. The filtered samples were then analyzed by capillary electrophoresis.

\section{Capillary electrophoresis analysis}

Capillary electrophoresis analysis was conducted using a capillary electrophoresis system model Photal CAPI-3300 (Otsuka Electronics. Co. Ltd., Osaka, Japan) equipped with a fused silica capillary of $75-\mu \mathrm{m}$ i.d. with a total length of $80 \mathrm{~cm}$ (effective length of $68 \mathrm{~cm}$ ). Before starting the analysis, the capillary was conditioned with $0.1 \mathrm{M} \mathrm{NaOH}$ for 5 min followed by conditioning with distilled water for $3 \mathrm{~min}$ and electrolyte buffer for $3 \mathrm{~min}$ (50 mM sodium phosphate buffer, $\mathrm{pH}$ 3.0). Between each run, the capillary was flushed with distilled water $(1 \mathrm{~min})$ and electrolyte buffer ( $3 \mathrm{~min}$ ). The temperature of the capillary was set at $25^{\circ} \mathrm{C}$ and then the samples and/or the authentic standards ( $\beta$-alanine betaine and TMA) were injected hydrostatically $(25 \mathrm{~mm}, 60 \mathrm{~s})$. During the run and in order to avoid sample carry-over into the electrophoresis buffer, the capillary was dipped twice in distilled water and washing buffer (same electrophoresis buffer that set in other tubes). The applied potential was $20 \mathrm{kV}$, and the peaks of TMA-salt and $\beta$-alanine betaine-ester were monitored at $262 \mathrm{~nm}$.

\section{Statistical analyses}

Statistical analyses were performed with the SPSS v. 18.0 software (SPSS Inc., Chicago, IL, USA). One-factor ANOVA was performed to identify statistically significant differences among treatments, followed by Tukey's HSD test $(P \leq 0.05)$.

\section{Results and discussion}

\section{Sample derivatization}

In the literature, it has been established that 4-bromophenacyl bromide reagent reacts with quaternary ammonium compounds and can accurately be used for their quantification (Gorham et al. 1982). We have adapted and modified this derivatization method for the determination of $\beta$-alanine betaine and TMA in plant and microbial samples by capillary electrophoresis. Since $\beta$-alanine betaine and TMA lack of useful chromophores that lead to the inability for detection at the UV/vis range, in the current work, they were derivatized with 4-bromophenacyl bromide to form $\beta$-alanine betaine-ester and TMA-salt (Figure 1). These reaction products showed a maximum absorption at $262 \mathrm{~nm}$, which was within the range 214 to $266 \mathrm{~nm}$ reported previously for various betaine esters (Gorham et al. 1982; Zhang et al. 2002). Determination of betaine as the 4-bromophenacyl ester has previously been reported to be exceptionally sensitive and specific (Gorham et al. 1982). Generally, absolute acetonitrile is usually used as solvent of 4-bromophenacyl for reaction with betaines (Gorham et al. 1982) giving a rapid esterification reaction at neutral or slightly alkaline $\mathrm{pH}$ levels. Nevertheless, in the current work, the reaction was carried out in less than $80 \%$ acetonitrile solution that gave an average yield of 4-bromophenacyl esters and salts of more than $70 \%$. To avoid the chemical decomposition of $\beta$-alanine betaine through $\mathrm{C}-\mathrm{N}$ bond cleavage that may lead to the production of trimethylamine and acrylate under alkaline condition (Gorham et al. 1982; Zhang et al. 2002), the derivatization condition was optimized and carried out at slightly acidic conditions ( $\mathrm{pH}$ 5.6) in the reaction mixture that contain potassium dihydrogen phosphate/ distilled water/acetonitrile $(1: 1: 4 v / v)$. Interestingly, these conditions resulted in efficient detection and quantification of $\beta$-alanine betaine in its intact form without decomposition as shown from the derivatized authentic standard of $\beta$-alanine betaine (Figure 2). In the meanwhile, TMA was also effectively detected and quantified after derivatization under the same conditions. Thus, the derivatization protocol of the current study could strikingly be used for simultaneous determination of both $\beta$-alanine betaine and TMA in plant, food, and microbial samples. In many previous reports, various betaines were esterified with 4-bromophenacyl bromide in the presence of a potassium bicarbonate/potassium dihydrogen phosphate/acetonitrile $(1: 1: 4 v / v)$ (Zhang et al. 1997; Nishimura et al. 2001; Zhang et al. 2002). Under these conditions, peak of trimethylamine salt derived from the breakdown of $\beta$-alanine betaine was observed in the capillary electropherogram as confirmed by using authentic trimethylamine (Nishimura et al. 2001; Zhang et al. 2002). During derivatization with 4-bromophenacyl bromide, the 

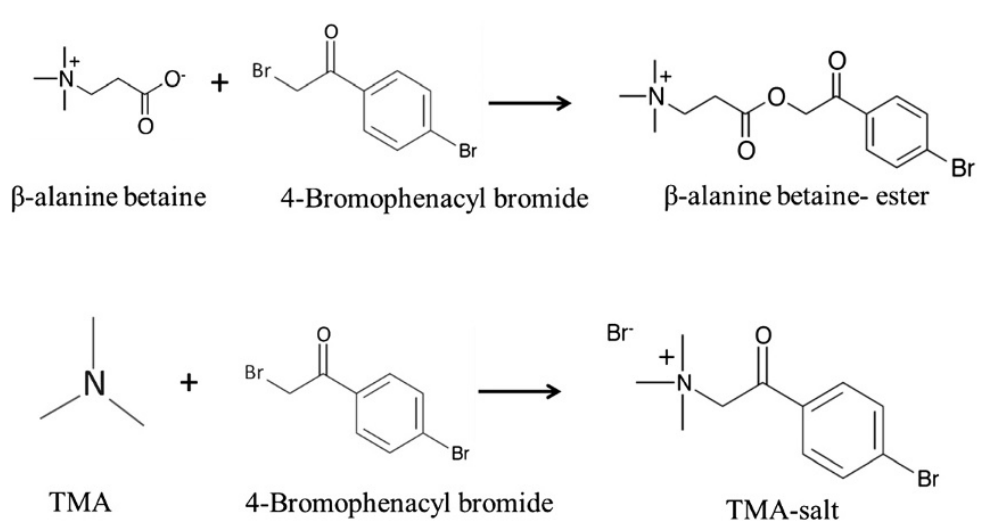

Figure 1 Reaction of $\beta$-alanine betaine and trimethylamine (TMA) with 4-bromophenacyl bromide and formation of $\beta$-alanine betaine-ester and TMA-salt.

compound dimethylsulfoniopropionate (DMSP), which structurally resembles $\beta$-alanine betaine, was also degraded to dimethylsulfide (DMS) and acrylate under an alkaline condition (Gorham et al. 1982; Zhang et al. 2005). Zhang et al. (2005) also reported that they kept the decomposition of DMSP as minimum by lowering the $\mathrm{pH}$ of the esterification reaction mixture to 4.0 by using 18 Crown-6 instead of potassium carbonate. In the current study, the decomposition of $\beta$-alanine betaine and consequently the production of the trimethylamine were completely avoided by omitting potassium bicarbonate from the derivatization buffer.

\section{Repeatability, linearity, and detection limit of TMA and} $\beta$-alanine betaine

The repeatability of the proposed method was explored by five consecutive runs of separate authentic standards $(2.5 \mathrm{mM})$ of TMA and $\beta$-alanine betaine. The relative standard deviation (RSD) for migration time of TMA and $\beta$-alanine betaine was $0.09 \%$ and $0.16 \%$, respectively.

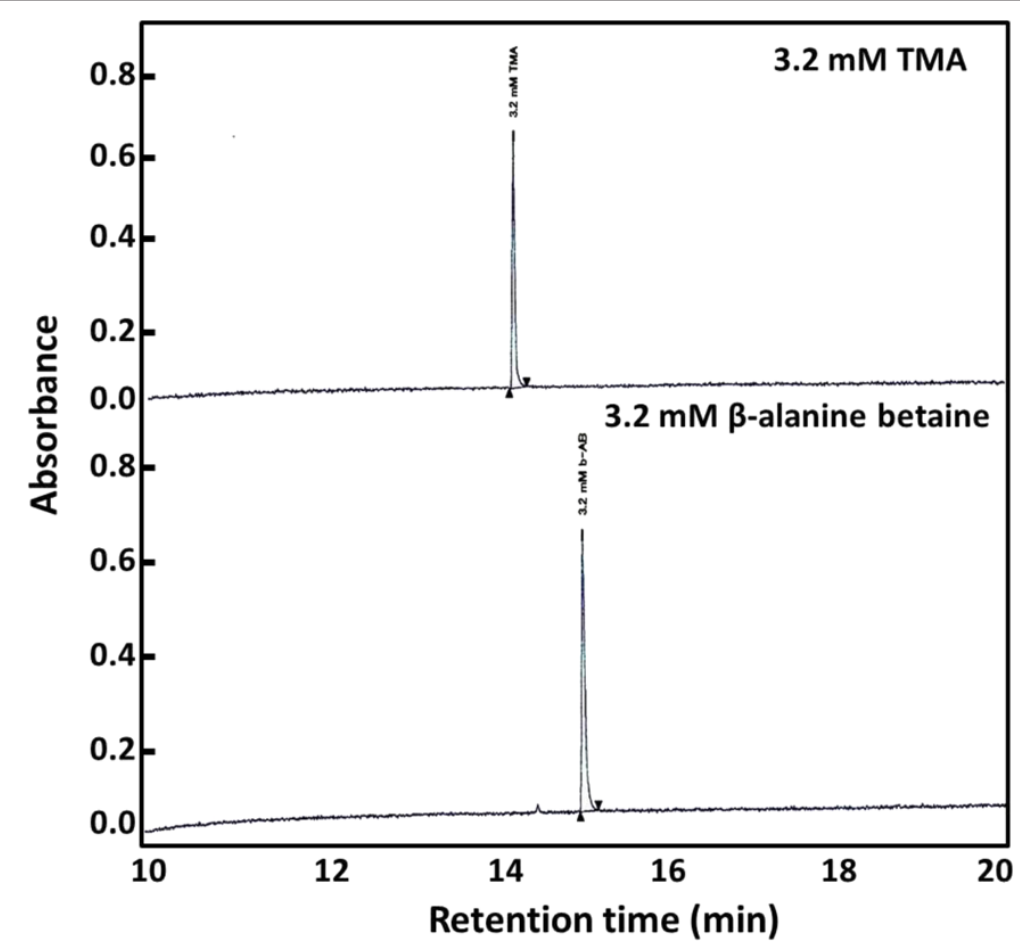

Figure 2 Authentic standard of TMA salt and $\beta$-alanine betaine ester detected by capillary electrophoresis. About $3.2 \mathrm{mM}$ of $\beta$-alanine betaine and TMA were esterified with 4-bromophenacyl bromide as described in the 'Methods' section. The samples were injected hydrostatically $(25 \mathrm{~mm}, 60 \mathrm{~s})$ at $25^{\circ} \mathrm{C}$. The applied potential was $20 \mathrm{kV}$, and the peaks were monitored at $262 \mathrm{~nm}$. 
While, the RSD for peak area of the analytes TMA and $\beta$-alanine betaine was $4.24 \%$ and $2.57 \%$, respectively. These values were in general agreement with those reported previously for TMA and glycine betaine when analyzed by capillary electrophoresis (Timm and Jørgensen 2002; Zhang et al. 2002). However, lower percentage of RSD of glycine betaine, choline, and TMA analyzed by ion exchange chromatography with non-suppressed conductivity detection method was recently reported (Zhang and Zhu 2007). To determine the day-to-day repeatability of the analysis, the samples were analyzed by capillary electrophoresis for three consecutive days, performed as sequence of five runs each day. The RSD of the migration time was $2.45 \%$ and $1.96 \%$ for TMA and $\beta$-alanine betaine, respectively, whereas that of the peak area was $3.49 \%$ and $3.74 \%$ for TMA and $\beta$-alanine betaine, respectively. In their analysis of day-to-day repeatability of capillary electrophoresis method for estimation of various amines, Timm and Jørgensen (2002) reported a RSD of less than $3 \%$ for migration time and less than $10 \%$ for peak area. They stated that these figures are acceptable in many applications; however, the percentage of RSD could be improved by including an internal standard within the samples. Linearity was investigated using the stock solution containing either TMA or $\beta$-alanine betaine, which was serially diluted. Then, eight concentrations $(0.05$ to $32 \mathrm{mM})$ of the analytes were analyzed in triplicate, and the calibration curves were constructed by plotting the peak area versus the concentration $(\mathrm{mM})$ of each analyte. The results showed that the suggested procedure produced highly linear calibration curves (Figure 3 ) with the correlation coefficients of 0.9933 and 0.9997 for TMA and $\beta$-alanine betaine, respectively. The linearity range of $\beta$-alanine betaine in the current study is greater than the range 0.05 to $5.0 \mathrm{mM}$ reported previously for many betaines analyzed by capillary electrophoresis (Zhang et al. 2002). Moreover, the linearity range of TMA in the current study is also greater than the range 0.25 to $10.0 \mu \mathrm{g} / \mathrm{ml}$ reported previously for the analysis of TMA in water samples by liquid chromatography
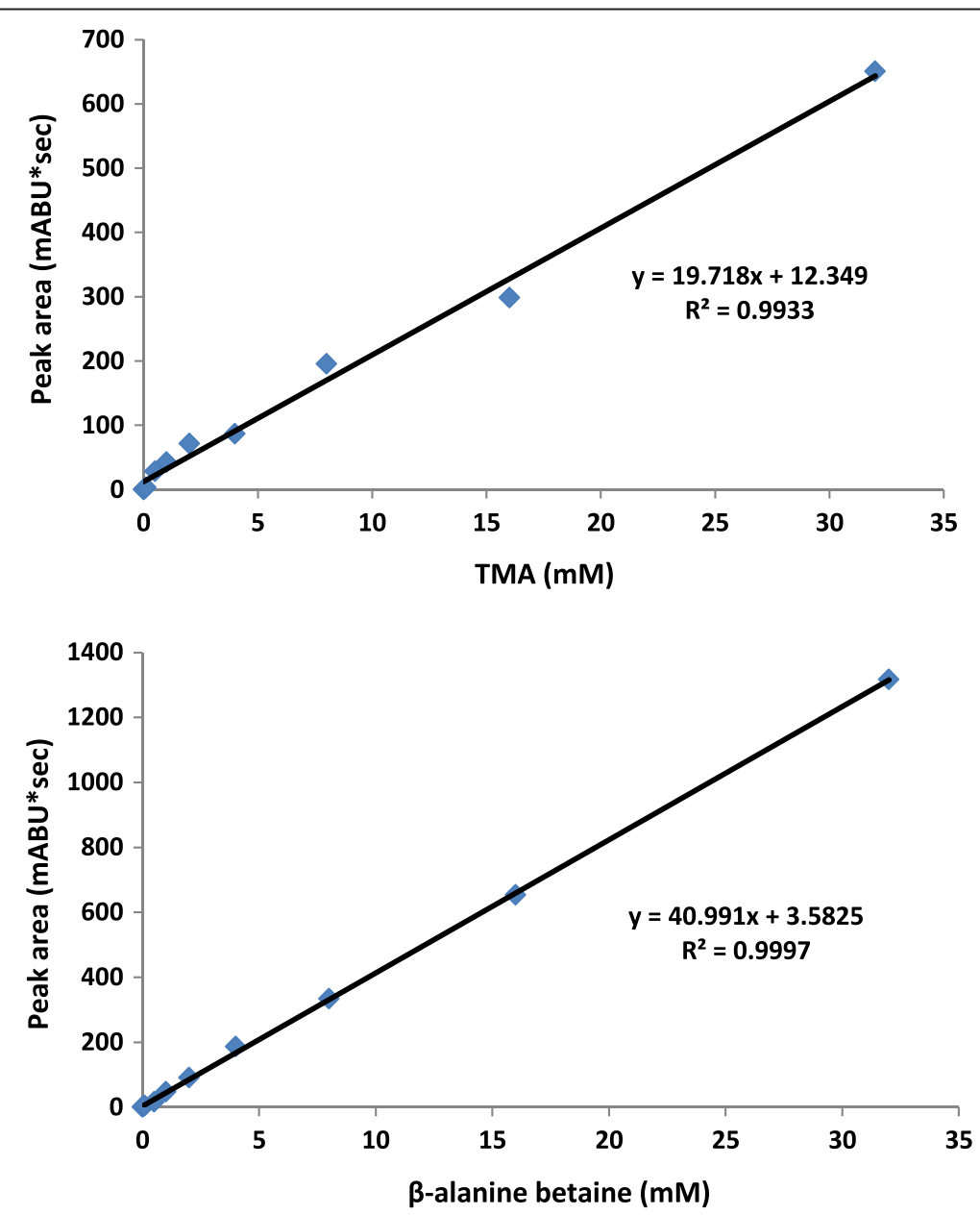

Figure 3 Linearity curves of $\beta$-alanine betaine and trimethylamine as estimated by the developed capillary electrophoresis method. Trimethylamine (upper panel); $\beta$-alanine betaine (lower panel). 


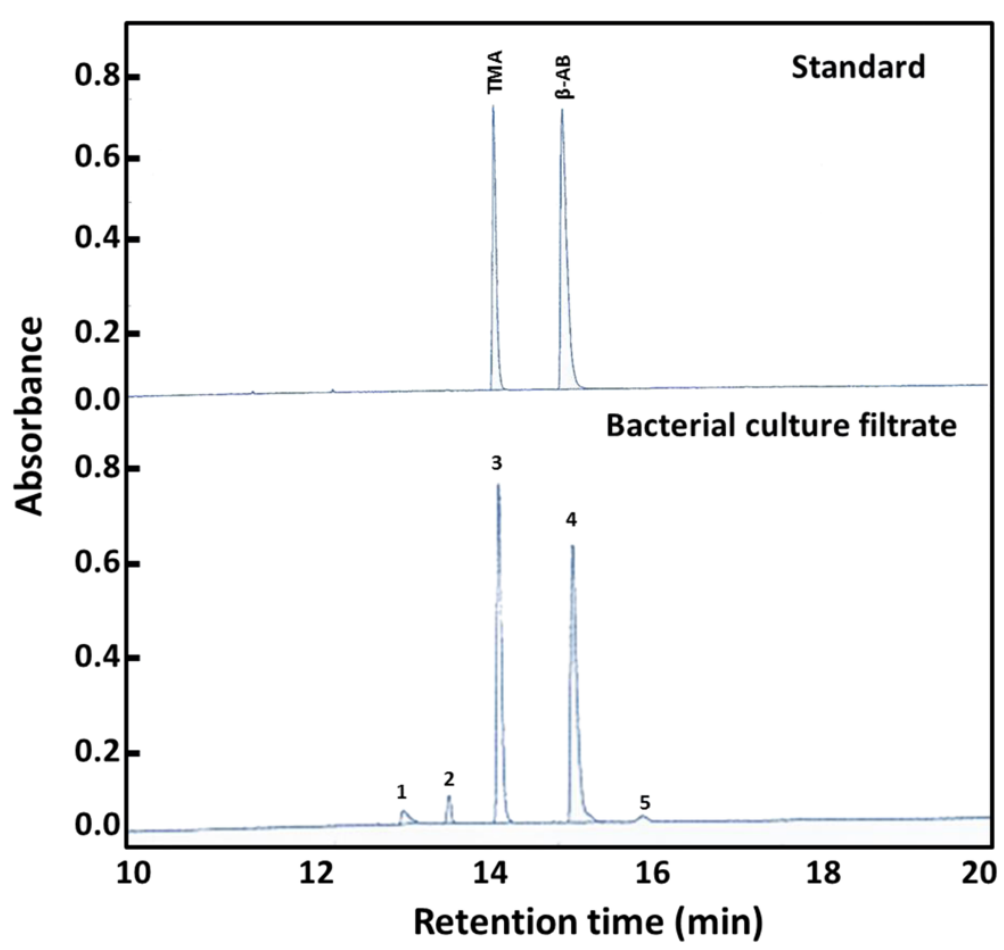

Figure $\mathbf{4}$ Capillary electrophoresis chromatogram of the intermediate metabolites of homocholine biodegradation by soil microorganism. The culture filtrate samples were esterified with 4-bromophenacyl bromide as described in the 'Methods' section. The samples were injected hydrostatically $(25 \mathrm{~mm}, 60 \mathrm{~s})$ at $25^{\circ} \mathrm{C}$. The applied potential was $20 \mathrm{kV}$, and the peaks were monitored at $262 \mathrm{~nm}$. The chromatogram showed the generated metabolite peaks from the degraded homocholine by the isolated strains alongside with authentic standard peaks of trimethylamine (TMA) and $\beta$-alanine betaine $(\beta-A B)$.

(Chafer-Pericas et al. 2004). The good linearity range of the current methods is a good indication for its applicability to accurately estimate these analytes in various samples including plants, microbial, food, and clinical samples. The limit of detection was calculated as the concentration that produced a signal-to-noise ratio of 3 and was estimated by analyzing solution of decreasing concentration of TMA and $\beta$-alanine betaine until this

Table 1 Trimethylamine and $\beta$-alanine betaine content in bacterial culture and plant samples

\begin{tabular}{lcc}
\hline Samples & $\begin{array}{l}\boldsymbol{\beta} \text {-alanine } \\
\text { betaine }\end{array}$ & Trimethylamine \\
\hline Bacterial culture (mmol/l) & & \\
Arthrobacter sp. strain E5 & 5.52 & 9.22 \\
Pseudomonas sp. strain A9 & 4.62 & 41.48 \\
Rhodococcus sp. strain A2 & 3.89 & 30.93 \\
Rhodococcus sp. strain A4 & 4.38 & 23.96 \\
Plant ( $\mu$ mol/g DW) & & \\
Limonium suffruticosum & 65.97 & 28.93 \\
Phragmites australis & 64.44 & 26.96 \\
Elaeagnus oxycarpa (200 mM NaCl) & 16.29 & n.d. \\
\hline
\end{tabular}

DW, dry weight; n.d., not detected. ratio was observed. The limit of detection of both compounds was found to be $0.01 \mathrm{mM}(100 \mu \mathrm{M})$. Similarly, the detection limit of glycine betaine estimated by low $\mathrm{pH}$ capillary electrophoresis method was reported to be $0.01 \mathrm{mM}$ (Nishimura et al. 2001; Zhang et al. 2002). Moreover, slightly lower detection limit $(0.005 \mathrm{mM})$ for DMSPester by low $\mathrm{pH}$ capillary electrophoresis has also been reported (Zhang et al. 2005). On the other hand, a lower detection limit $(50 \mathrm{ng} / \mathrm{ml})$ of TMA in water samples analyzed by liquid chromatography has also been reported (Chafer-Pericas et al. 2004). However, the detection limit of many amines including trimethylamine as analyzed by capillary electrophoresis with indirect UV-detection mode was reported to be $0.01 \mathrm{mM}$ (Timm and Jørgensen 2002). Generally, the detection limits of both compounds by the proposed method in the current study well agreed with those of the previously reported detection methods for individual compounds. Strikingly, in the current method, both compounds could simultaneously be determined even if they exist in relatively lower concentrations. Although, the proposed method may be suitable for most applications concerning the determination of TMA and $\beta$-alanine betaine in plants, microbial, environmental, and food samples, methods with extremely lower detection limits for these analytes might still be required. 
Application of the method to microbial and plant samples To illustrate an application of the developed method, three samples of either microbial cultures or dry cell reaction mixtures or plant leaves were prepared as described in the 'Methods' section. The intermediate metabolites in the microbial culture filtrate of different microorganisms grown on homocholine as the sole source of carbon and nitrogen was analyzed by the developed method. During the consumption of homocholine by the growing cell cultures of the isolated strains of the genera Arthrobacter, Pseudomonas, and Rhodococcus (Mohamed Ahmed et al. 2009a, b; Mohamed Ahmed et al. 2010), there were a concurrent formation and accumulation of some soluble metabolites identified as trimethylamine (peak 3, TMA) and $\beta$-alanine betaine (peak $4, \beta-A B$ ) as detected by capillary electrophoresis method (Figure 4). Under the optimized derivatization conditions of the developed method, the TMA-salt and $\beta$-alanine betaine-ester were successfully separated and detected with very clear and sharp peaks using a UV detector at $262 \mathrm{~nm}$. The amounts of these metabolites were successfully estimated (Table 1) by calculating the area of each peak. It can be seen that the quantity of TMA in all microbial culture samples was higher compared to the quantity of $\beta$-alanine betaine. This is an ideal phenomenon because these strains were observed to cleave $\mathrm{C} / \mathrm{N}$ pond of $\beta$-alanine betaine and rapidly use the resulted carbon chain as source of carbon with the release of TMA as a major metabolite (Mohamed Ahmed et al. 2009a, b; Mohamed Ahmed et al. 2010; Mohamed Ahmed et al. 2014). To check the accuracy of this method, the TMA concentration in the culture filtrate and intact cell reaction products of the isolated strains was analyzed by using picric acid-based colorimetric method (Dyer 1945). The results showed no significant differences from that obtained by the developed capillary electrophoresis method. To expand the applicability of the developed method, the plant leaves that were obtained from Xinjiang, China, in August 2010 were also prepared and analyzed by this method as described in the 'Methods' section. The results again showed clear peaks of TMA and $\beta$-alanine betaine in the leaf samples of many of plants growing on saline soil in this area (Figure 5). These osmotolerant plants accumulated sufficient amounts of nitrogenous compounds to cope with these osmotic

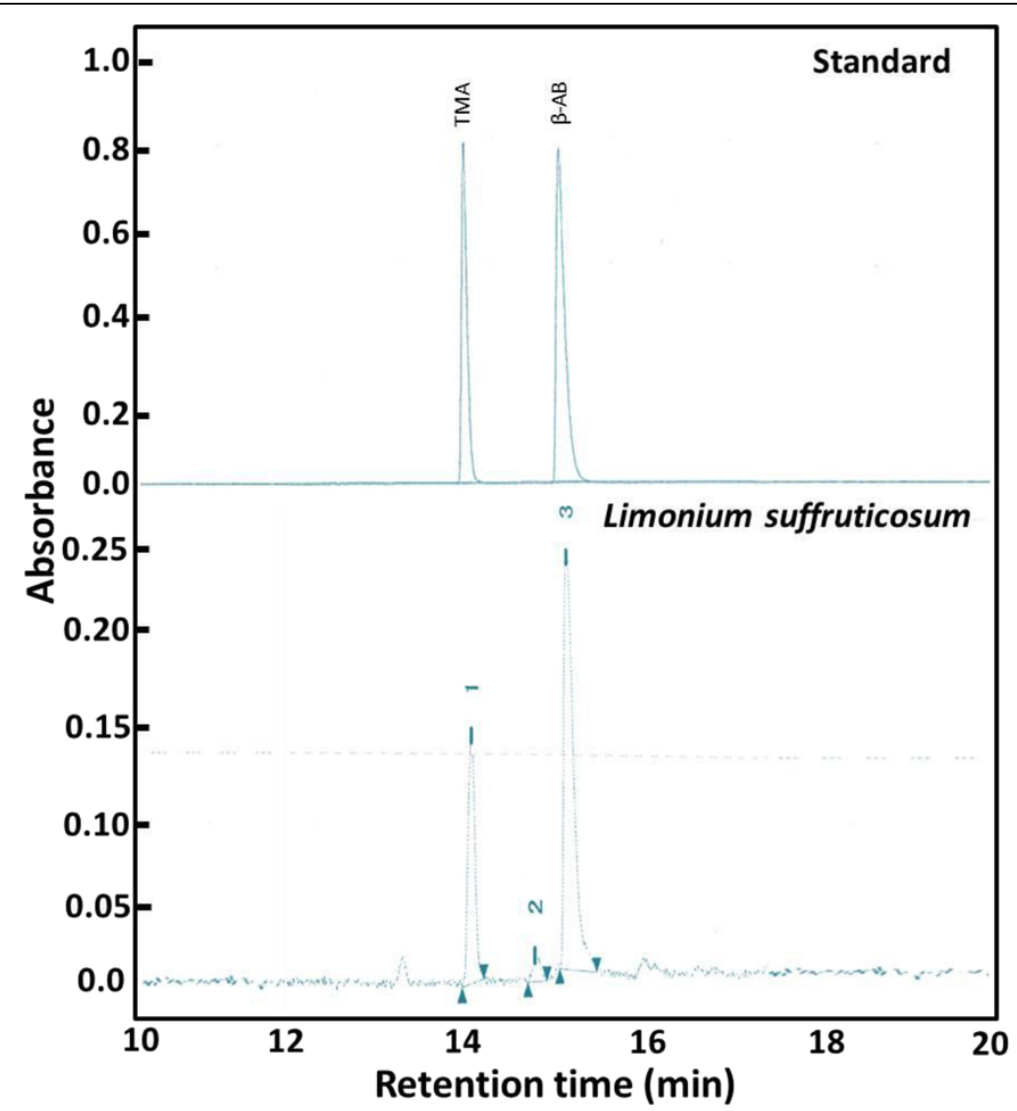

Figure 5 Capillary electrophoresis chromatogram of the osmolytes in the leaves of Limonium suffruticosum grown in saline soil. Plant leaf samples were esterified with 4-bromophenacyl bromide as described in the 'Methods' section. The samples were injected hydrostatically $(25 \mathrm{~mm}, 60 \mathrm{~s})$ at $25^{\circ} \mathrm{C}$. The applied potential was $20 \mathrm{kV}$, and the peaks were monitored at $262 \mathrm{~nm}$. The chromatogram showed the accumulated osmolytes in the plant samples alongside with authentic standard peaks of trimethylamine (TMA) and $\beta$-alanine betaine ( $\beta$-AB). 
stresses. Of these nitrogenous compounds, considerable amounts of both TMA and $\beta$-alanine betaine were quantitatively estimated using the developed method (Table 1). Similarly, the concentration of $\beta$-alanine betaine in many plants of the family Plumbaginaceae was found in the range of 1 to $147 \mu \mathrm{g} / \mathrm{g} \mathrm{DW}$, which was estimated by either TLC and autoradiography (Rathinasabapathi et al. 2000) or ${ }^{1} \mathrm{H}$ NMR (Baysalfurtana et al. 2013). Collectively, the above findings clearly demonstrated the suitability of the developed method for the simultaneous detection and quantification of these analytes in microbial culture and plant samples. Although it is not tested in the current study, the developed method could efficiently be used for the estimation of TMA and $\beta$-alanine betaine in both food and feed samples.

\section{Conclusion}

A capillary electrophoresis method for the simultaneous determination of TMA and $\beta$-alanine betaine was developed. The method described here has generally wide detection range suitable for analysis of TMA and $\beta$-alanine betaine in microbial and plant samples. The advantages of the current method are its low cost, low detection limit, simple operation, rapid, and high sensitivity.

\section{Competing interests}

The authors declare that they have no competing interests.

\section{Authors' contributions}

IAMA has performed most of the experimental and analytical work and prepared the draft of the manuscript. AM has performed the plant-related parts of the experimental work with the direct help from IAMA. The guidelines and supervision of this work was provided by NM, NY, and $\pi$. All authors read and approved the final manuscript.

\section{Acknowledgements}

Financial assistance from the Ministry of Education, Culture, Sports, Science, and Technology of Japan in the form of a scholarship for the first and second authors is gratefully acknowledged.

\section{Author details}

${ }^{1}$ Department of Food Science and Technology, Faculty of Agriculture, University of Khartoum, Shambat 13314, Sudan. ${ }^{2}$ Arid Land Research Center, Tottori University, 1390 Hamasaka, Tottori 680-0001, Japan. ${ }^{3}$ School of Agricultural, Biological, and Environmental Sciences, Faculty of Agriculture, Tottori University, Koyama, Tottori 680-8553, Japan.

Received: 3 June 2014 Accepted: 24 July 2014

Published online: 22 August 2014

\section{References}

Baysalfurtana G, Duman H, Tipirdamaz R (2013) Seasonal changes of inorganic and organic osmolyte content in three endemic Limonium species of Lake Tuz (Turkey). Turk J Bot 37:455-463

Blunden G, El Barouni MM, Gordon SM, McLean WFH, Rogers DJ (1981) Extractions, purification and characterization of Dragendorff-positive compounds from some British marine algae. Botanica Marina 24:451-456

Chafer-Pericas C, Herraez-Hernandez R, Campins-Falco P (2004) Liquid chromatographic determination of trimethylamine in water. J Chromatogr A 1023:27-31

Craciun S, Balskus EP (2012) Microbial conversion of choline to trimethylamine requires a glycyl radical enzyme. Proc Natl Acad Sci USA 109:21307-21312

Dalgaard P (2006) Microbiology of marine muscle foods. In: Hui YH (ed) Handbook of food science, technology and engineering. CRC, Boca Raton, pp. $53-1-53-20$
Dyer WJ (1945) Amines in fish muscles 1. Colorimetric determination of trimethylamine as the picrate salt. J Fish Res Board Can 6(5):351-358 Ghaly AE, Dave D, Budge S, Brooks MS (2010) Fish spoilage mechanisms and preservation techniques: review. Amer J Appl Sci 7:859-877

Gorham J (1995) Betaines in higher plants-biosynthesis and role in stress metabolisms. In: Wallsgrove RM (ed) Amino acids and their derivatives in higher plants. Cambridge University Press, Cambridge, England, pp 173-203

Gorham J, McDonnell E, Wyn Jones RG (1982) Determination of betaines as ultraviolet-absorbing esters. Anal Chim Acta 138:277-283

Grieve CM, Grattan SR (1983) Rapid assay for determination of water soluble quaternary ammonium compounds. Plant Soil 70:303-307

Hanson AD, Rathinasabapathi B, Rivoal J, Burnet M, Dillon MO, Gage DA (1994) Osmoprotective compounds in the Plumbaginaceae: a natural experiment in metabolic engineering of stress tolerance. Proc Natl Acad Sci USA 91:306-310

Mohamed Ahmed IA, Jiro A, Ichiyanagi T, Sakuno E, Mori N (2009a) Isolation and characterization of 3-N-trimethylamino-1-propanol degrading Rhodococcus sp. strain A2. FEMS Microbiol Lett 296(2):219-225

Mohamed Ahmed IA, Jiro A, Ichiyanagi T, Sakuno E, Mori N (2009b) Isolation and characterization of 3-N-trimethylamino-1-propanol degrading Arthrobacter sp. strain E5. Res J Microbiol 4(2):49-58

Mohamed Ahmed IA, Jiro A, Ichiyanagi T, Sakuno E, Mori N (2010) Isolation and characterization of homocholine degrading Pseudomonas sp. strain A9 and B9b. World J Microbiol Biotechnol 26(8):1455-1464

Mohamed Ahmed IA, Eltayeb MM, Arima J, Mori N, Yamanaka N, Taniguchi T (2014) Screening for enzymatic activities in the degradation pathway of homocholine by soil microorganisms. Australian J Bas Appl Sci 8(2):222-233

Nishimura N, Zhang J, Abo M, Okubo A, Yamazaki S (2001) Application of capillary electrophoresis to the simultaneous determination of betaines in plants. Anal Sci J 17:103-106

Rathinasabapathi B, Sigua C, Ho J, Gage DA (2000) Osmoprotectant $\beta$-alanine betaine synthesis in the Plumbaginaceae: S-adenosyl-L-methionine dependent $\mathrm{N}$ methylation of $\beta$-alanine to its betaine via $\mathrm{N}$-methyl and N, N-dimethyl $\beta$-alanines. Physiol Plant 109:225-231

Rhodes D, Hanson AD (1993) Quaternary ammonium and tertiary sulfonium compounds in higher plants. Annu Rev Plant Physiol Plant Mol Biol 44:357-384

Shintani H, Polonsky J (1997) Handbook of capillary electrophoresis applications. Blackie Academic \& Professional, London

Siminhoff ML, Burke JF, Saukkonen JJ, Ordinario AT, Doty R, Dunn S (1977) Biochemical profile of uremic breath. The New Engl J Med 297:132-135

Timm M, Jørgensen BM (2002) Simultaneous determination of ammonia, dimethylamine, trimethylamine and trimethylamine-N-oxide in fish extracts by capillary electrophoresis with indirect UV-detection. Food Chem 76(4):509-518

Yancey PH (2005) Organic osmolytes as compatible, metabolic and counteracting cytoprotectants in high osmolarity and other stresses. J Exp Biol 208:2819-2830

Zhang J, Zhu Y (2007) Determination of betaine, choline and trimethylamine in feed additive by ion-exchange liquid chromatography/non-suppressed conductivity detection. J Chromatogr A 1170:114-117

Zhang J, Okubo A, Yamazaki S (1997) Determination of betaines in plants by low-pH capillary electrophoresis as their phenacyl esters. Bunseki Kagaku (in Japanese) 46:275-279

Zhang J, Nishimura N, Okubo A, Yamazaki S (2002) Development of an analytical method for the determination of betaines in higher plants by capillary electrophoresis at low pH. Phytochem Anal 13:189-194

Zhang J, Nagahama T, Abo M, Okubo A, Yamazaki S (2005) Capillary electrophoretic analysis of dimethylsulfoniopropionate in sugarcane and marine algal extracts. Talanta 66:244-248

doi:10.1186/s40543-014-0038-x

Cite this article as: Mohamed Ahmed et al: Simultaneous determination of $\beta$-alanine betaine and trimethylamine in bacterial culture and plant samples by capillary electrophoresis. Journal of Analytical Science and Technology 2014 5:38 